\title{
Abstract \\ Increasing the quality and value of involving simulated patients in simulation-based education, research and practice
}

\author{
S. Gough, L. Greene, A. Natali, R. Mackinnon, S. Roberts, M. Hellaby, A. \\ Whitcombe, B. Webster, N. Tuttle, D. Nestel
}

Relevance: Simulated Patients (SPs) are volunteers, actors, service users or students trained to portray the role of patients or relatives in healthcare education [1]. Despite their involvement in healthcare education programmes, no standardised, evidence-based approach to training SPs in how to portray roles [2] or deliver constructive feedback as part of the debrief $[1,3]$ existed in the UK. Furthermore, there was no framework to support organisations to plan and implement SP involvement in education, research and practice in the UK [3].

Purpose: The purpose of these projects was to develop, pilot and evaluate a standardised, evidence-based training programme for SP Trainers and SPs in the North West of England.

The aims were to develop:

- A bespoke Simulated Patient Train-The-Trainer blended learning package (SP3 T) for Simulation Trainers

- $\quad$ An innovative Train-The-Simulated Patient (2TSP) blended learning package incorporating performing arts pedagogy for SPs

- A framework, checklist and instructional guide for organisations, to enable them to implement and embed the SP Programme within their workplace

- Guidance for 'Trained SPs' to volunteer in simulation-based education (SBE) activities, via the 'SP Passport' and 'Trained SP' database

Approach/evaluation: Firstly, the literature was explored, then a regional survey was undertaken. Eighty-nine participants responded from 24 different organisations across 4 Universities and 20 Hospital Trusts in the North West of England. Findings from the literature review and survey were used to develop the 'Simulated Patient Programme' and related resources. The programme incorporates the SP3T and 2TSP blended learning packages. Each package consists of pre-requisite e-learning, followed by an interactive workshop. The SP Programme [3,5], SP Common Framework [4], Instructional Guide [5], SP Database [5] and SP Passport [5] were developed and then piloted and evaluated by 18 SP Trainers and 34 SPs.

\section{Outcomes:}

- The survey identified variances in SP terminology, recruitment, payment, contracts, risk assessment and training, which were considered when developing the SP Programme and SP Common Framework. Training methods varied; over $1 / 3$ of respondents were unsure of whether their organisation offered SP training. Challenges relating to recruitment and quality assurance of SP involvement were identified. 
- A standardised, evidence-based SP Programme incorporating innovative techniques has been produced. Resources enabled organizations the ability to plan, develop, integrate, deliver and evaluate SP involvement.

- Valuable involvement of service users, who advised on the design and delivery of the SP Programme, plus a robust evaluation, has enabled the development of high quality, appropriate resources.

- $\quad$ The Instructional Guide firmly embeds the SP Common Framework and SP Checklist; enabling organizations to successfully prepare for SP engagement.

- SP Database testing confirmed usability in organisations with diverse IT infrastructures.

- The SP Passport was reported to be a valuable resource to track, record and monitor SP training and involvement in SBE.

Discussion and conclusions: The SP Programme is currently being rolled out throughout the North West of England, to ensure as many people as possible benefit from the project and resources.

Impact and implications: It is envisaged that engagement with well-trained SPs will enhance SBE, improve education for the future and existing health and social care workforce and ultimately lead to improvements in patient safety.

Funding acknowledgement: The Simulated Patient Project was funded by NHS Health Education North West (NHS HENW). 